\title{
Publicaciones científicas en revistas peruanas de psicología: un análisis desde la participación estudiantil
}

\author{
Ronald M. Hernández \\ Universidad San Ignacio de Loyola, Lima, Perú \\ https://orcid.org/0000-0003-1263-2454 \\ Renzo Felipe Carranza Esteban \\ Universidad Peruana Unión, Tarapoto, Perú \\ https://orcid.org/0000-0002-4086-4845 \\ Tomás Caycho-Rodríguez* \\ Universidad Privada del Norte, Lima, Perú \\ https://orcid.org/0000-0002-5349-7570 \\ Isabel Cabrera-Orosco \\ Universidad Continental, Huancayo, Perú \\ https://orcid.org/0000-0002-0375-2879 \\ Dennis Arias Chávez ${ }^{1}$ \\ Universidad Continental, Arequipa, Perú. \\ https://orcid.org/0000-0003-1500-8366
}

\section{Resumen}

El estudio describe la producción científica de estudiantes de psicología en revistas peruanas de psicología registrados en el directorio Latindex, en el año 2017. Se realizó un estudio teórico analizando 220 artículos de 12 revistas de psicología. Se excluyó todo aquel tipo de publicación que no cuente con la estructura IMRYD. Se registraron 485 autores con afiliación nacional e internacional de los cuales el 8.25\% son estudiantes de psicología, los cuales han participado en la publicación de 29 artículos (investigaciones originales, revisiones y estudios de caso). De los 29 artículos con autoría estudiantil, el 64.5\% son artículos del área clínica y el 35.5\% del área educativa. El tipo de investigación con mayor predominancia es instrumental (54.8\%). Se concluye que la producción científica de los estudiantes de psicología de pregrado en las revistas de psicología es baja. Esto hace necesario fortalecer e incentivar la generación y divulgación de los estudios científicos psicológicos por parte de los estudiantes en el Perú. Palabras clave: estudiante universitario; servicio bibliográfico; revistas de psicología; investigación psicológica.

\section{Scientific publications in peruvian journals of psychology: an analysis from student participation}

\section{Abstract}

The study describes the scientific production of psychology students in Peruvian Psychology Journals registered in the Latindex directory in 2017. A theoretical study that analyzed 220 articles from 12 journals of psychology was carried out. Any publication 
that did not have the IMRAD structure was excluded. There were 485 authors with national and international affiliation, of which 8.25\% were psychology students and had participated in the publication of 29 articles (original research, reviews, and case studies). Of the 29 articles with student authorship, $64.5 \%$ were articles from the clinical area and $35.5 \%$ from the educational area. The most prevalent type of research was instrumental (54.8\%). It is concluded that the scientific production of undergraduate psychology students in psychology journals is low. This makes it necessary to strengthen and encourage the generation and dissemination of psychology scientific studies by students in Peru.

Keywords: university student, bibliographic service, psychology journals, psychology research

\title{
Publicações científicas em revistas peruanas de psicologia: uma análise desde a participação dos estudantes
}

\begin{abstract}
Resumo
O estudo descreve a produção científica de estudantes de psicologia em revistas peruanas dessa disciplina, cadastrados no diretório Latindex, em 2017. Realizou-se um estudo técnico por meio da análise de 220 artigos de 12 revistas de psicologia. Excluiu-se todo tipo de publicação que não contasse com a estrutura IMRYD. Dos 485 autores com filiação nacional einternacional cadastrados, 8,25\% eram estudantes de psicologia, os quais tinham participado na publicação de 29 artigos (pesquisas originais, revisões e estudos de caso). Dos 29 artigos de autoria estudantil, 64,5\% correspondiam a artigos da área clínica e 35,5\% pertenciam à área educativa. $O$ tipo de pesquisa de maior predominância foi a instrumental (54,8\%). Conclui-se que a produção cientifica dos estudantes de psicologia dos cursos de graduação presente nas revistas de psicologia é baixa. Isto torna necessário fortalecer e incentivar a geração e difusão dos estudos científicos psicológicos de estudantes no Peru.

Palavras-chaves: estudante universitário; serviço bibliográfico; revistas de psicologia; pesquisa psicológica.
\end{abstract}

Como citar:

Hernandez, R., Carranza, F., Caycho-Rodríguez, T., Cabrera-Orozco, I., \& Arias-Chávez, D. (2019). Publicaciones científicas en revistas peruanas de psicología: un análisis desde la participación estudiantil. Revista Digital de Investigación en Docencia Universitaria, 13(2), 19-28. https://doi.org/10.19083/ridu.2019.1082

\section{L} a investigación es el eje de la formación profesional en cualquier carrera universitaria (Gutiérrez, \& Mayta, 2003). Dentro de este proceso, la investigación formativa favorece a que el estudiante desarrolle competencias necesarias para un aprendizaje permanente en investigación (Miyahira, 2009). En las carreras profesionales relacionadas con la salud, la investigación es cada vez más reconocida como un componente importante dentro de los planes de estudios de pregrado (Amgad, Tsui, Liptrott, \& Shash, 2015) y un factor determinante para investigar en la vida profesional (Reinders, Kropmans \& Cohen-Schotanus, 2005). En este sentido, realizar investigación en pregrado permite contar con mejores profesionales de la salud, que realicen una lectura más crítica de la literatura y la evidencia científica. Además, llevar a cabo un proyecto de investigación y presentarlo en una conferencia o publicarlo como un artículo de investigación, genera una sensación de logro que motivará al estudiante para llevar su investigación a un nivel más alto (Cheung, 2018).

La publicación de los resultados de un trabajo de investigación en revistas indexadas es el indicador más importante para valorar la producción científica de un país (Huamaní, Chávez-Solís, Domínguez-Haro, \& Solano-Aldana, 2007; Reyes, Alarcón \& Bahamón, 2014). Esto ha generado que se promueva en los estudiantes de pregrado, de diversas carreras profesionales de salud, llevar a cabo proyectos de investigación y presentar sus 
resultados en conferencias y revistas científicas (Cheung, 2018). Sin embargo, estudios realizados en países latinoamericanos con alta y baja producción científica, reportan que aún es limitada la cantidad de estudiantes de carreras de Ciencias de la Salud que publican sus trabajos de investigación en revistas indexadas (Barbón \& Bascó, 2016; Corrales-Reyes, Rodríguez, Reyes, \& García, 2017).

Estudios indican que las publicaciones de artículos de investigación realizados por estudiantes en revistas médicas peruanas representan alrededor del $5 \%$ del total de publicaciones, donde el $70 \%$ de los artículos estudiantiles se concentran solo en las facultades de medicina de la Universidad Nacional Mayor de San Marcos y Universidad Peruana Cayetano Heredia (Huamaní, Chávez-Solis, \& Mayta-Tristán, 2008; Romaní, \& Wong, 2009). Otro estudio señala que en el periodo 2003 y 2009 se realizaron 110 trabajos de investigación como parte de un curso de Epidemiología en una universidad peruana, pero solo el $11.8 \%$ de los mismos fue publicado en alguna revista biomédica (Alarcón-Villaverde, Romaní, \& Gutiérrez, 2010).

En Colombia, el 10\% de los artículos científicos publicados en una revista médica, tienen participación estudiantil (Pachajoa-Londoño, 2006). En Venezuela, solo el 3.9\% de 333 artículos originales revisados en el periodo 2001 al 2005 contaron con participación estudiantil como autores (Angulo, Angulo, Huamani, \& Mayta-Tristán, 2008). Finalmente, un estudio sobre la producción estudiantil médica en revistas en español en el 2011 señaló que solo el 3.6\% de los 2.476 artículos originales identificados contaron con estudiantes de medicina como autores, donde el Perú era uno de los países con mayor cantidad de publicaciones estudiantiles, junto con Colombia y Chile (Taype-Rondán, Palma-Gutiérrez, Palacios-Quintana, Carbajal-Castro, \& Ponce-Torres, 2014).

Esta realidad de los países latinoamericanos es diferente a lo que ocurre en el contexto europeo o de Estados Unidos. En los Países Bajos, de un total de 2973 estudiantes, el 14.5\% publicó por lo menos un artículo científico durante los últimos 3 años (Van Eyk, et al. 2010), mientras que, en Alemania, el 66\% de estudiantes de medicina publicaron artículos en revistas científicas (Cursiefen, \& Altunbas, 1998). Finalmente, en Estados
Unidos, un $25 \%$ de los estudiantes de medicina realizaron publicaciones científicas (Zier, Friedman, \& Smith, 2006).

Como se observa existen algunos estudios que analizan la producción científica de los estudiantes de carreras de ciencias de la salud, principalmente en el ámbito de la medicina. Debido a la importancia de la publicación estudiantil, y al no encontrar reportes acerca de la productividad científica de estudiantes de psicología en el Perú, se realizó el presente estudio que tuvo como objetivo describir la producción científica de los estudiantes de psicología en las revistas indizadas en Latindex. Los resultados tendrán importantes implicancias prácticas ya que permitirá identificar posibles errores y sugerir acciones de mejora para la formación en investigación de los estudiantes de psicología de pregrado en el Perú.

\section{Método}

\section{Población}

El estudio de tipo teórico (Montero \& León, 2005) consideró como unidad de análisis a 220 artículos en formato PDF o HTML (texto completo), 12 volúmenes y 25 números publicados en 12 revistas peruanas de psicología incluidas en el Directorio Latindex, además deberían contar con una visibilidad web en una plataforma o portal de revistas y presentar continuidad de publicación hasta el año 2017 (Tabla 1). Del total de artículos identificados y de acuerdo con los criterios de inclusión; se consideraron solo artículos originales, revisiones y estudios de caso. Se definió como artículos con participación estudiantil aquellos en los cuales al menos uno de los autores mencionó en su afiliación ser estudiante de pregrado. No se consideraron reseñas, cartas al editor, ensayos, entrevistas, editoriales ni obituarios.

\section{Instrumento}

Se utilizó un formato de recolección de información en MS Excel que incluía variables como: tipo de artículo, tipo de investigación, nombre de la revista donde se publicó el artículo, tipo de autor (profesional o estudiante), sexo del autor, datos de afiliación institucional y país de procedencia. 


\section{Procedimiento}

La búsqueda de los artículos se realizó en 3 fases. En la primera, se buscó de manera general las revistas cuyo registro indicaban el subtema de psicología. En la segunda fase, se ingresó de manera manual a cada portal de revistas para identificar los artículos originales, de revisión y estudios de caso, lo que permitió elaborar una data por afiliación institucional. Finalmente, se buscó por autor (estudiante o profesional), y para identificar y estar seguros de que el artículo era presentado por estudiantes; se buscaron las palabras: estudiante, no graduado, pregrado.

Los datos recolectados fueron tabulados y analizados con el paquete estadístico Stata versión 15 y para reportar las tablas de frecuencias se utilizó el programa Microsoft Excel 2010.

Tabla 1.

Revistas científicas peruanas de psicología y sus características de publicación.

\begin{tabular}{|c|c|c|c|c|}
\hline $\begin{array}{c}\text { Revistas } \\
\text { de Psicología }\end{array}$ & Institución & $\begin{array}{c}\text { Año } \\
\text { de inicio }\end{array}$ & Indización & $\begin{array}{c}\text { Frecuencia } \\
\text { de publicación }\end{array}$ \\
\hline \multirow{5}{*}{ Revista de Psicología } & \multirow{5}{*}{$\begin{array}{l}\text { Pontificia Universidad Católica } \\
\text { del Perú }\end{array}$} & \multirow{5}{*}{1983} & Scopus & \multirow{5}{*}{ Semestral } \\
\hline & & & SciELO & \\
\hline & & & Redalyc & \\
\hline & & & Dialnet & \\
\hline & & & Latindex & \\
\hline \multirow{5}{*}{ Liberabit } & \multirow{5}{*}{ Universidad San Martín de Porres } & \multirow{5}{*}{1995} & SciELO & \multirow{5}{*}{ Semestral } \\
\hline & & & WoS (ESCI) & \\
\hline & & & Redalyc & \\
\hline & & & Dialnet & \\
\hline & & & Latindex & \\
\hline \multirow{5}{*}{$\begin{array}{l}\text { Propósitos y } \\
\text { Representaciones }\end{array}$} & \multirow{5}{*}{$\begin{array}{l}\text { Universidad San Ignacio de } \\
\text { Loyola }\end{array}$} & \multirow{5}{*}{2013} & SciELO & \multirow{5}{*}{ Semestral } \\
\hline & & & WoS (ESCI) & \\
\hline & & & Redalyc & \\
\hline & & & Dialnet & \\
\hline & & & Latindex & \\
\hline $\begin{array}{l}\text { Revista de Psicología y } \\
\text { Trabajo Social }\end{array}$ & $\begin{array}{l}\text { Universidad Inca Garcilaso } \\
\text { de la Vega }\end{array}$ & 2012 & Latindex & Semestral \\
\hline \multirow{3}{*}{ Persona } & \multirow{3}{*}{ Universidad de Lima } & \multirow{3}{*}{1998} & Redalyc & \multirow{3}{*}{ Anual } \\
\hline & & & Dialnet & \\
\hline & & & Latindex & \\
\hline Avances en Psicología & $\begin{array}{l}\text { Universidad Femenina del } \\
\text { Sagrado Corazón }\end{array}$ & 1993 & Latindex & Semestral \\
\hline $\begin{array}{l}\text { Revista de Investigación } \\
\text { de Estudiantes de } \\
\text { Psicología JANG }\end{array}$ & $\begin{array}{l}\text { Universidad César } \\
\text { Vallejo }\end{array}$ & 2002 & Latindex & Semestral \\
\hline $\begin{array}{l}\text { Revista de Investigación } \\
\text { en Psicología }\end{array}$ & $\begin{array}{l}\text { Universidad Nacional Mayor de } \\
\text { San Marcos }\end{array}$ & 1998 & Latindex & Semestral \\
\hline Revista Psicoanálisis & Sociedad Peruana de Psicoanálisis & 1999 & Latindex & Semestral \\
\hline \multirow{2}{*}{ Interacciones } & \multirow{2}{*}{$\begin{array}{l}\text { Instituto Peruano de Orientación } \\
\text { Psicológica }\end{array}$} & \multirow{2}{*}{2015} & Dialnet & \multirow{2}{*}{ Trimestral } \\
\hline & & & Latindex & \\
\hline Revista Digital EOS Perú & Instituto Digital EOS Perú & 2013 & Latindex & Semestral \\
\hline $\begin{array}{l}\text { Revista de Psicología } \\
\text { (Arequipa) UCSP }\end{array}$ & Universidad Católica San Pablo & 2011 & Latindex & Semestral \\
\hline
\end{tabular}




\section{Resultados}

Se encontró un total de 485 autores de los cuales 40 son estudiantes de psicología (38 peruanos y 2 colombianos). Respecto a la participación estudiantil por sexo, se encontró 28 varones y $12 \mathrm{mu}$ jeres. Se observa en la Tabla 2 que existe mayor predominancia de publicación estudiantil hacia los artículos originales.

Con relación a los autores por país (tanto estudiantes como no estudiantes), se encontró que 288 son peruanos (59.5\%), 57 de México (11.8\%), 50 de Argentina (10.3\%), seguido de otros países. En relación solo a los autores estudiantiles por país,
Perú es el país que mayor destaca con autoría, seguido de Colombia. Los resultados con más detalle pueden observarse en la tabla 3.

Respecto a la afiliación institucional, se identifica a la Universidad César Vallejo, como la que registra mayor número de autores (37.5\%) (Tabla 4).

Finalmente, de los 29 artículos con presencia estudiantil (estudiantes de psicología), el 64.5\% fueron artículos del área clínica (Tabla 5). Además, el tipo de investigación con mayor predominancia fue instrumental (54.8\%) (Tabla 6); mientras que la revista con mayor participación estudiantil es la Revista de Investigación de Estudiantes de Psicología JANG (Tabla 7).

Tabla 2.

Tipos de artículos publicados.

\begin{tabular}{|l|c|c|c|c|}
\hline & \multicolumn{2}{|c|}{ Total } & \multicolumn{2}{c|}{ Estudiantes } \\
\hline & $f$ & $\%$ & $n$ & 12.3 \\
\hline Artículos originales & 153 & 70 & 27 & 0.5 \\
\hline Estudio de Caso & 5 & 2 & 1 & 0.5 \\
\hline Revisiones & 13 & 6 & 1 & - \\
\hline Otros & 49 & 22 & - & $\%$ \\
\hline
\end{tabular}

Tabla 3.

Autores estudiantiles con afiliación institucional por país.

\begin{tabular}{|c|c|c|c|c|}
\hline \multirow{2}{*}{ Países } & \multicolumn{2}{|c|}{ Total de autores } & \multicolumn{2}{|c|}{ Autores estudiantiles } \\
\hline & $f$ & $\%$ & $f$ & $\%$ \\
\hline Perú & 288 & 59.5 & 38 & 95 \\
\hline México & 57 & 11.8 & - & - \\
\hline Argentina & 50 & 10.3 & - & - \\
\hline Brasil & 21 & 4.3 & - & - \\
\hline España & 17 & 3.5 & - & - \\
\hline Colombia & 11 & 2.3 & 2 & 5 \\
\hline Bélgica & 7 & 1.4 & - & - \\
\hline Chile & 7 & 1.4 & - & - \\
\hline Ecuador & 6 & 1.2 & - & - \\
\hline Estados Unidos & 5 & 1.0 & - & - \\
\hline Portugal & 4 & 0.8 & - & - \\
\hline Costa Rica & 3 & 0.6 & - & - \\
\hline Israel & 3 & 0.6 & - & - \\
\hline Italia & 1 & 0.2 & - & - \\
\hline Paraguay & 1 & 0.2 & - & - \\
\hline Suiza & 1 & 0.2 & - & - \\
\hline Guatemala & 1 & 0.2 & - & - \\
\hline Alemania & 1 & 0.2 & - & - \\
\hline Total & 485 & 100 & 40 & 100 \\
\hline
\end{tabular}


Tabla 4.

Autores estudiantiles según afiliación institucional.

\begin{tabular}{|c|c|c|c|}
\hline Universidades (afiliación) & Tipo de universidad & $f$ & $\%$ \\
\hline Universidad Metropolitana (Colombia) & Privada & 2 & 5 \\
\hline Universidad de Lima & Privada & 2 & 5 \\
\hline Universidad César Vallejo & Privada & 15 & 37.5 \\
\hline Universidad Nacional Mayor de San Marcos & Estatal & 18 & 45 \\
\hline \multirow[t]{2}{*}{ Universidad Católica San Pablo } & Privada & 3 & 7.5 \\
\hline & & 40 & 100 \\
\hline
\end{tabular}

Tabla 5.

Artículos con participación estudiantil según área de la psicología.

\begin{tabular}{|c|c|c|}
\hline Áreas de la psicología & $\boldsymbol{f}$ & $\boldsymbol{\%}$ \\
\hline Clínica & 20 & 68.9 \\
\hline Educativa & 9 & 31.1 \\
\hline Organizacional & - & - \\
\hline Social & - & - \\
\hline Total & $\mathbf{2 9}$ & $\mathbf{1 0 0}$ \\
\hline
\end{tabular}

Tabla 6.

Artículos con participación estudiantil según tipo de investigación

\begin{tabular}{|c|c|c|}
\hline Tipos de investigación & $\boldsymbol{f}$ & $\boldsymbol{\%}$ \\
\hline Descriptivo & 4 & 13.8 \\
\hline Descriptivo - Correlacional & 10 & 34.4 \\
\hline Instrumental & 15 & 51.8 \\
\hline Total & $\mathbf{2 9}$ & 100 \\
\hline
\end{tabular}

Tabla 7.

Cantidad de autores estudiantiles por revista.

\begin{tabular}{|l|c|}
\multicolumn{1}{|c|}{ Revista } & $f$ \\
\hline Revista de Psicología PUCP & - \\
\hline Liberabit & - \\
\hline Propósitos y Representaciones & 2 \\
\hline Revista de Psicología y Trabajo Social & - \\
\hline Persona & 2 \\
\hline Avances en Psicología & - \\
\hline Revista de Investigación de Estudiantes de Psicología JANG & 15 \\
\hline Revista de Investigación en Psicología UNMSM & 12 \\
\hline Revista Psicoanálisis & - \\
\hline Interacciones & 6 \\
\hline Revista Digital EOS Perú & - \\
\hline Revista de Psicología (Arequipa) UCSP & 3 \\
\hline Total & 40 \\
\hline
\end{tabular}




\section{Discusión}

La universidad tiene una misión formativa y otra investigativa. Actualmente formar estudiantes investigadores es de vital importancia (Christensen \& Eyring, 2011), pues la investigación dentro de la educación superior debe comenzar durante el pregrado (Castro-Rodríguez, 2015). Como parte de este desafío, las universidades presentan planes de investigación formativa pues es necesario que las mallas curriculares contemplen estrategias para la elaboración y desarrollo de trabajos de investigación y su posible publicación (Carrizo, 2010).

Los estudiantes son agentes activos en su formación y aprendizaje y centran su atención y energía en dominar aquellas tareas que le permitan reforzar su labor de investigación, estableciendo redes de apoyo con adultos y sus pares (Wang, \& Eccles, 2012). Por lo tanto, la participación de los estudiantes es fundamental en la producción de conocimientos, en este caso, concretados en productos de alto impacto como son las revistas científicas (Appleton, Christenson \& Furlong, 2008; Castro, Sihuay-Torres \& Pérez-Jiménez, 2018).

La implementación de investigaciones y la publicación de resultados son fundamentales para un estudiante de psicología. Si los resultados de los estudios de investigación o las documentaciones de los programas no se publican, otros investigadores no pueden apreciar el valor de la evidencia generada, y la ciencia en general no podría progresar. En este sentido, y con el fin de promover la producción científica, el Sistema Nacional de Evaluación y Acreditación enfatiza que las escuelas profesionales deben contar con revistas que permitan a los estudiantes difundir los resultados de sus investigaciones (Carranza \& Turpo, 2018).

Los resultados de este estudio son comparables al $11.8 \%$ de artículos originales escritos por estudiantes durante el periodo 2003 y 2009 en una universidad peruana (Alarcón-Villaverde et al., 2010), y superior al $10 \%$ reportado en Colombia (Pachajoa-Londoño, 2006) y al 3.9\% en Venezuela entre el 2001 al 2005 (Angulo et al., 2008). Esta limitada producción científica también se observa en el ámbito de los psicólogos profesionales peruanos. Por ejemplo, del total de artículos publica- dos en la Revista Latinoamericana de Psicología, entre 1994 y 2014 (1026 artículos), solo 6 son de autores peruanos, lo que representa el 0.8\% (Arias, Ceballos \& Arpasi, 2015). Otros estudios bibliométricos más actuales brindan nuevas evidencias acerca de la pobre producción científica de los psicólogos peruanos (Morgado-Gallardo et al., 2018; Salas, et al., 2018).

Existen diferentes factores académicos y de investigación que están asociados a los resultados anteriores, como la falta de acceso de información adecuada y el apoyo de asesores con experiencia en investigación, que son factores importantes, y a tomar en cuenta, al momento de planificar y realizar una investigación (Atamari-Anahui, Sucasaca-Rodríguez, \& Marroquin-Santa Cruz, 2016; Castro-Rodríguez, 2015; Mamani, 2018, Mamani, 2019).

Dentro de las limitaciones para la publicación estudiantil se encuentra también la falta de valoración del trabajo realizado, la ausencia en el país de una cultura de divulgación; la escasa participación en las publicaciones a pesar de que existe evidencia de la organización de congresos estudiantiles nacionales e internacionales; además, existe deficiente consigna en los datos de afiliación, ya que, en muchos casos, los autores colocan sus instituciones mas no su formación académica. En este sentido, resulta valioso capacitar a los estudiantes en la publicación de sus investigaciones, incluyendo cursos o talleres en la formación universitaria.

Los resultados de predominancia en autoría peruana pueden deberse a que la población analizada incurre a revistas editadas por instituciones nacionales. Otros estudios, que evaluaron la producción de científica de diferentes países, revelan que los países que tuvieron más revistas con publicaciones estudiantiles fueron Colombia, Chile y Perú (Taype-Rondán et al., 2014; Carvajal, 2018).

Respecto a la participación estudiantil según tipo de estudio, los resultados indicaron que la mayoría son estudios instrumentales. Esto difiere con los hallazgos de Castro-Rodríguez (2015) quien encontró que el $6.4 \%$ de los artículos publicados por estudiantes de ciencias de la salud fueron ensayos clínicos, el $16.6 \%$ estudios experimentales, el 13.9\% artículos de revisión y el 63.1\% correspondió a artículos derivados de estudios observacionales. Al respecto Mamani (2019) reporta 
que el mayor porcentaje de tesis presentadas por estudiantes de universidad privada de Lima son correlacionales (81.2\%), mientras que solo 4 (2.7\%) son instrumentales.

El estudio presenta como limitación metodológica que se consideró la producción científica estudiantil realizada en el año 2017. Esto impide visualizar en retrospectiva y prospectiva el desarrollo de la investigación en psicología de los estudiantes peruanos.

Se concluye que la producción científica de los estudiantes de pregrado psicología es baja; los artículos publicados son principalmente son del tipo instrumental, y el mayor porcentaje se concentra en una revista estudiantil. Por ello, una manera de motivar a los estudiantes para publicar los resultados de sus investigaciones radica en aumentar las horas dedicadas a investigar y retribuir a los estudiantes que logran difundir los resultados de sus investigaciones en forma de artículo científico. Para facilitar esto, las revistas deben brindar una mejor orientación y promover la discusión de las investigaciones.

\section{Referencias}

Alarcón-Villaverde, J., Romaní, F., \& Gutiérrez, C. (2010). Publicaciones científicas estudiantiles producidas en el curso de Epidemiología de la Facultad de Medicina de la Universidad Nacional Mayor de San Marcos durante el periodo 2003-2009. Anales de la Facultad de Medicina, 71(2), 111-116. https://doi. org/10.15381/anales.v71i2.82

Amgad, M., Tsui, M. M. K., Liptrott, S. J., \& Shash, E. (2015). Medical student research: an integrated mixed-methods systematic review and meta-analysis. PLoS one, 10(6), e0127470. https://doi. org/10.1371/journal.pone.0127470

Angulo, R., Angulo, F., Huamani, C., \& Mayta-Tristán, P. (2008). Publicación Estudiantil en Revistas Médicas Venezolanas, 2001-2005. Revista Ciencia e Investigación Médico Estudiantil Latinoamericana, 13(1), 6-8. Recuperado de http://www.cimel.felsocem.net/ index.php/CIMEL/article/view/156

Appleton, J., Christenson, S., \& Furlong, M. (2008). Stu- dent engagement with school: Critical conceptual and methodological issues of the construct. Psychology in the Schools, 45(5), 369-386. https://doi. org/10.1002/pits.20303

Arias, W. L., Ceballos, K. D., \& Arpasi, M. S. (2015). El aporte de los psicólogos peruanos en la Revista Latinoamericana de Psicología de 1994 al 2014: un estudio bibliométrico. Revista Peruana de Psicología y Trabajo Social, 4(1), 57-76.

Atamari-Anahuia, N., \& Sucasaca-Rodríguez, C., \& Marroquin-Santa Cruz, J. (2016). Publicación científica de asesores de tesis de pregrado en una escuela de medicina de Cusco, Perú. Investigación en Educación Médica, 5(20), 279-280. Recuperado de https://www. redalyc.org/articulo.oa?id=3497/349747925011

Barbón, O., \& Bascó, E. (2016). Clasificación de la actividad científica estudiantil en la educación médica superior. Educación Médica, 17(2), 55-60. https://doi. org/10.1016/j.edumed.2016.02.001

Christensen, C., \& Eyring, H. (2011) The Innovative University: Changing the DNA of Higher Education from the Inside Out. Journal of Research \& Method in Education, 5(1), 1-4.

Carranza, R., \& Turpo, J. (2018). Limitantes de la producción y publicación científica universitaria. Educación Mé dica. https://doi.org/10.1016/j.edumed.2017.12.002

Carrizo, J. (2010). Importancia de la investigación en la formación de pregrado. Panorama Cuba y Salud, 5(3), 3-4. Recuperado de http://revpanorama.sld.cu/index.php/panorama/article/viewFile/98/pdf

Carvajal, A. (2018). Análisis bibliométrico de la participación estudiantil en publicaciones de artículos científicos en revistas de ciencias de la salud indizadas en SciELO Bolivia, periodo 2010-2016. Gaceta Médica Boliviana, 41(1), 31-35. Recuperado de http:// www.gacetamedicaboliviana.com/index.php/gmb/ article/view/169/0

Castro-Rodríguez, Y. (2015). Perfil bibliométrico de la producción científica en la Revista Kiru. Evaluación del intervalo 2005-2014. KIRU, 12(2), 65-69. Recuperado de http://www.usmp.edu.pe/odonto/servicio/2015/ Kiru_12-2_v_p64-68.pdf

Castro, Y., Sihuay-Torres, K., \& Pérez-Jiménez, V. (2018). Producción científica y percepción de la investigación por estudiantes de odontología. Educación Médica, 19(1), 19-22. https://doi.org/10.1016/j. edumed.2016.11.001

Cheung, B. M. (2018). Medical student research: is it necessary and beneficial? Postgraduate medical journal 
94(1112), 317-317. https://doi.org/10.1136/postgradmedj-2018-135834

Corrales-Reyes, I., Rodríguez, M., Reyes, J., \& García, M. (2017). Limitantes de la producción científica estudiantil. Educación Médica, 18(3), 199-202. https:// doi.org/ 10.1016/j.edumed.2016.11.005

Cursiefen, C., \& Altunbas, A. (1998). Contribution of medical student research to the MedlineTM-indexed publications of a German medical faculty. Medical Education, 32(4), 439-440. https://doi.org/10.1046/ j.1365-2923.1998.00255.x

Gutiérrez, C., \& Mayta, P. (2003). Publicación desde el Pre-Grado en Latinoamérica: importancia, limitaciones y alternativas de solución. CIMEL Ciencia e Investigación Médica Estudiantil Latinoamericana, 8(1), 54-60. Recuperado de http://200.62.146.19/ BVRevistas/cimel/v08_n1/PDF/a10v8n1.pdf

Huamaní, C., Chávez-Solís, P., Domínguez-Haro, W., \& Solano-Aldana, M. (2007). Producción científica estudiantil: análisis y expectativas. Revista Peruana de Medicina Experimental y Salud Pública, 24(4), 44446. https://doi.org/10.17843/rpmesp.2007.244.1146

Huamaní, Ch., Chávez-Solis, P., \& Mayta-Tristán, P. (2008). Aporte estudiantil en la publicación de artículos científicos en revistas médicas indizadas en Scielo-Perú, 1997 - 2005. Anales de la Facultad de Medicina, 69(1), 42-45. https://doi.org/10.15381/anales. v69i1.1182

Mamani, O. (2018). El asesor de tesis como Coach: una alternativa para impulsar la producción científica estudiantil. Educación Médica Superior, 33(1). Recuperado de http://ems.sld.cu/index.php/ems/article/ view/1590/761

Mamani, O. (2019). Publicación científica y características de asesores de tesis de una escuela de psicología peruana. Apuntes Universitarios, 9(2), 27-36. https:// doi.org/10.17162/au.v9i2.356

Miyahira, J. (2009). La investigación formativa y la formación para la investigación en el pregrado. Revista Médica Herediana, 20(3), 119-122. https://doi. org/10.20453/rmh.v20i3.1010

Montero, I., \& León, O. G. (2005). Sistema de clasificación del método en los informes de investigación en Psicología. International Journal of clinical and health psychology, 5(1), 115-127.

Morgado-Gallardo, K., Salas, G., Faúndez, M.J., López-López, W., Ventura-León, J., Barboza-Palomino, ... Guerra-Labbé, L. (2018). 25 años de Suma Psicológica. Un análisis bibliométrico. Suma Psicológica, 25(2), 90 -
101. https://doi.org/10.14349/sumapsi.2018.v25.n2.1. Pachajoa-Londoño, H. M. (2006). Publicación de artículos originales desde el pregrado en una revista médica colombiana entre 1994-2004. CIMEL Ciencia e Investigación Médica Estudiantil Latinoamericana, 11(1), 24-26. Recuperado de http://200.62.146.19/ BVRevistas/cimel/v11_n1/pdf/a08.pdf

Reinders, J. J., Kropmans, T. J., \& Cohen-Schotanus, J. (2005). Extracurricular research experience of medical students and their scientific output after graduation. Medical Education, 39(2), 237-237. https://doi. org/10.1111/j.1365-2929.2004.02078.x

Reyes, L., Alarcón, Y., \& Bahamón, M. (2014). Tendencias en las líneas de investigación de Doctorados en Psicología de universidades iberoamericanas. Psicogente, 17(32), 442-451. Recuperado de http://revistas.unisimon.edu.co/index.php/psicogente/article/ view/1456

Romaní, F., \& Wong, P. (2009). Reporte y serie de casos en el Perú: situación de un tipo de publicación subestimada. Análisis de las revistas médicas peruanas indizadas en SciELO-Perú, 1997-2008. Revista peruana de epidemiología, 13(3). Recuperado de http://200.62.146.19/BVRevistas/epidemiologia/ v13_n3/pdf/a09v13n3.pdf

Salas, G., Ravelo-Contreras, E. L., Mejía, S., Andrades, R., Acuña, E., Espinoza, F., ... \& Pérez-Acosta, A. M. (2018). Dos décadas de Acta Colombiana de Psicología: un análisis bibliométrico. Acta Colombiana de Psicología, 21(2), 13-25. https://doi.org/10.14718/ acp.2018.21.2.2

Taype-Rondán, A., Palma-Gutiérrez, E., Palacios-Quintana, M., Carbajal-Castro, C., \& Ponce-Torres, C. (2014). Producción científica estudiantil en Latinoamérica: un análisis de las revistas médicas de habla hispana indizadas en SciELO, 2011. FEM. Revista de la Fundación Educación Médica, 17(3), 171-177. https://doi. org/10.4321/S2014-98322014000300007

Van Eyk, H. J., Hooiveld, M. H., Van Leeuwen, T. N., Van der Wurff, B. L., De Craen, A. J., Dekker, F. W., \& NVMO-Special Interest Group on Scientific Education. (2010). Scientific output of Dutch medical students. Medical teacher, 32(3), 231-235. https://doi. org/10.3109/01421591003596592

Wang, M. T., \& Eccles, J.S. (2012). Social support matters: Longitudinal effects of social support on three dimensions of school engagement from middle to high school. Child Development, 83(3), 877-895. https://doi.org/10.1111/j.1467-8624.2012.01745.x 
Zier, K., Friedman, E., \& Smith, L. (2006). Supportive programs increase medical students' research interest and productivity. Journal of Inves tigative Medicine, 54(4), 201-207. https://doi. org/10.2310/6650.2006.05013 